\title{
Subthreshold Kaons Would Reveal Density Isomers
}

\author{
C. Hartnack, ${ }^{1,2}$ J. Aichelin, ${ }^{1}$ H. Stöcker ${ }^{3}$ and W. Greiner ${ }^{3}$ \\ ${ }^{1}$ Laboratoire de Physique Nucléaire de Nantes, F-44072 Nantes Cedex 03, France \\ ${ }^{2}$ Gesellschaft für Schwerionenforschung, D-64220 Darmstadt, Federal Republic of Germany \\ ${ }^{3}$ Institut für Theoretische Physik, Universität Frankfurt, D-60054 Frankfurt, Federal Republic of Germany
}

(Received 22 September 1993; revised manuscript received 7 April 1994)

\begin{abstract}
If density isomers exist they can be detected by measuring the excitation function of subthreshold kaon production. When the system reaches the density where the density isomer has influence on the equation of state (which depends on the beam energy and on the optical potential), we observe a jump in the cross section of the kaons whereas other observables change little. Above threshold $\bar{\Lambda}$ 's or $\bar{p}$ 's may be used to continue the search. This is the result of microscopic Boltzmann-UehlingUhlenbeck calculations.
\end{abstract}

PACS numbers: $25.70 .-\mathrm{z}, 21.65 .+\mathrm{f}, 25.75 .+\mathrm{r}$

One of the main motivations for the study of heavy ion collisions at energies in between $50 \mathrm{MeV}$ and several $\mathrm{GeV}$ is to determine the nuclear equation of state (EOS) as a function of density and temperature. Microscopic calculations have revealed the dependence of several observables on the EOS [1-3]. They include the transverse momentum transfer (flow), the enhanced emission of particles perpendicular to the reaction plane (squeeze), and the production of subthreshold kaons. This opens the possibility to determine the EOS by experiments.

In these calculations two parametrizations of the potential energy of the nucleons have been employed which mark the expected boundaries of the range of values. They are given by

$$
V(\rho)=\alpha\left(\frac{\rho}{\rho_{0}}\right)+\beta\left(\frac{\rho}{\rho_{0}}\right)^{\gamma}[\mathrm{MeV}],
$$

with $\alpha=-62,-178, \beta=23.5,140$, and $\gamma=2,7 / 6$ for the hard and soft EOS, respectively.

This form of the potential energy has the advantage of depending only on three parameters out of which two are fixed by the condition that in nuclear matter the energy per nucleon has a minimum of $-16 \mathrm{MeV}$ at normal nuclear matter density. It is convenient to express the third parameter in terms of the compressibility $K$ at normal nuclear matter density. For the soft EOS we obtain $K=200 \mathrm{MeV}$, for the hard EOS, $K=380 \mathrm{MeV}$.

They have the disadvantage of being not very realistic. It is well known that the nuclear potential is momentum dependent. The momentum dependence has been measured in $p$-nucleus collisions; the extension into the complete $\rho$-momentum plane is rather unknown. Guided by $G$-matrix calculations the best guess is presently a linear dependence on the density. Taking these considerations into account we can obtain a momentum dependent potential which gives the same compressibility at zero temperature as a hard or soft EOS [4]:

$$
\begin{aligned}
V\left(\rho, \mathbf{p}_{1}, \mathbf{p}_{2}\right)= & \alpha\left(\frac{\rho}{\rho_{0}}\right)+\beta\left(\frac{\rho}{\rho_{0}}\right)^{\gamma} \\
& +1.57 \ln ^{2}\left[c\left(\mathbf{p}_{1}-\mathbf{p}_{2}\right)^{2}+1\right] \frac{\rho}{\rho_{0}}[\mathrm{MeV}]
\end{aligned}
$$

with $c=5 \times 10^{-4} \mathrm{MeV}^{-2}$ and $\alpha=-65,-195, \beta=$ 19,149 , and $\gamma=2.09,1.14$ for the hard and soft EOS, respectively.

However, the nuclear EOS may not be that simple. It has been argued by several authors [5] that it may have a second minimum at densities higher than $2 \rho_{0}$. This can be caused by nonlinear scalar meson terms in the Lagrangian or by collective excitations of zero frequency spin-isospin modes in nuclear matter, called pion condensation. However, many of these models had problems in describing normal nuclear matter. This problem has been solved in more elaborate relativistic mean field models using an extended Walecka Lagrangian [6] with third and fourth power terms in the scalar field $[7,8]$. Relativistic mean field calculations with $\Delta$ resonances $[9,10]$ yield also a second minimum at about $\rho=3 \rho_{0}$. Its exact position and depth depend on the scalar and vector coupling constants of the deltas.

Until recently, due to the large experimental error bars, there was no evidence that the simple three parameter EOS is not sufficient to describe the experimental results. Recently the first high precision experiments have been performed at the SIS facility at GSI. They produced a puzzling result. The $4 \pi$ collaboration has measured the transverse momentum transfer as a function of the transverse energy and found that a reasonable agreement between the microscopic calculation and data can only be obtained if the EOS is hard [11]. Calculations with a soft equation of state are clearly off the data. The KAOS collaboration, which measured the production of subthreshold kaons at energies around $1 \mathrm{GeV}$, has found that even a soft equation of state underpredicts the observed yield. A hard equation of state would yield an additional suppression by a factor of $2[12,13]$. We would like to mention two other microscopic approaches, both using seemingly the same relativistic transport theory $[14,15]$. Whereas Ref. [14] agrees with our conclusions, Ref. [15] obtains a kaon yield which is 1 order of magnitude higher and therefore leads to completely different conclusions. A detailed discussion of these approaches may be found in [16]. 
Motivated by the theoretical interest in density isomers and by the seemingly contradictory experimental results, which leave room for new phenomena, we have investigated how the presence of density isomers would influence the observables.

In this Letter we will show that above a threshold energy density isomers change the kaon yield by 1 order of magnitude but leave other observables like the flow more or less unchanged. Below the threshold energy the density isomer now has influence. Thus we observe a jump in the excitation function. The energy at which this jump occurs depends on the density at which the isomer is located. This is the result of microscopic BoltzmannUehling-Uhlenbeck (BUU/VUU) calculations. The details of the numerical solution can be found in Ref. [1]. We would like to mention that in this approach the effective mass does not affect the collision term, i.e., the $\sqrt{s}$ is calculated with the free masses.

Figure 1 displays the three different equations of state we use in our calculations. The parameters of the momentum dependent interactions are chosen in a way that they reproduce for zero temperature the curves for their static counterparts. Up to a threshold which is higher than $2 \rho_{0}$ all these EOS's are identical and follow the hard equation of state [Eq. (1)]. The EOS's with a density isomer (Hisom) start to deviate from the hard EOS at $2.4 \rho_{0}$ (Hisom 1) and 2.6 $\rho_{0}$ (Hisom 2). The density isomer has a depth of $2 \mathrm{MeV}$ at $\rho=\rho_{\text {thres }}+0.3 \rho_{0}$. Above $\rho_{\text {thres }}+0.6 \rho_{0}$ we have

$$
E / N_{\text {Hisom }}\left(\rho / \rho_{0}\right)=E / N_{\mathrm{H}}\left(\rho / \rho_{0}-0.6\right)
$$

Before coming to the results of the calculation it is instructive to discuss qualitatively our expectations. We start with the production of kaons. Below threshold kaon production is concentrated at central collisions where the density is high. At $1 \mathrm{GeV} \mathrm{Au}+\mathrm{Au}$ the average density at the point of the kaon production is around $2 \rho_{0}$, the maximal value about $3 \rho_{0}[17]$. Therefore, the kaons test the high density zone itself. There is a sufficient number of collisions that some baryons can gain a substantial increase of their momenta which is necessary to overcome the threshold. The Fermi motion alone is not able to provide the additional required energy. Most of the kaons are produced in a two step process $[15,16,18]$,

$$
N_{1}+N_{2} \rightarrow N_{1}+\Delta, \quad N_{3}+\Delta \rightarrow N_{3}+\Lambda+K .
$$

Because of kinematical reasons this channel is much more effective than the channel

$$
\pi+N \rightarrow \Lambda+K
$$

which counts for not more than $20 \%$ of the produced kaons [19]. A decreasing mean free path of the $\Delta$ therefore enhances the kaon production because it reduces the probability that the $\Delta$ decays before it collides with another nucleon. A softening of the equation of state at

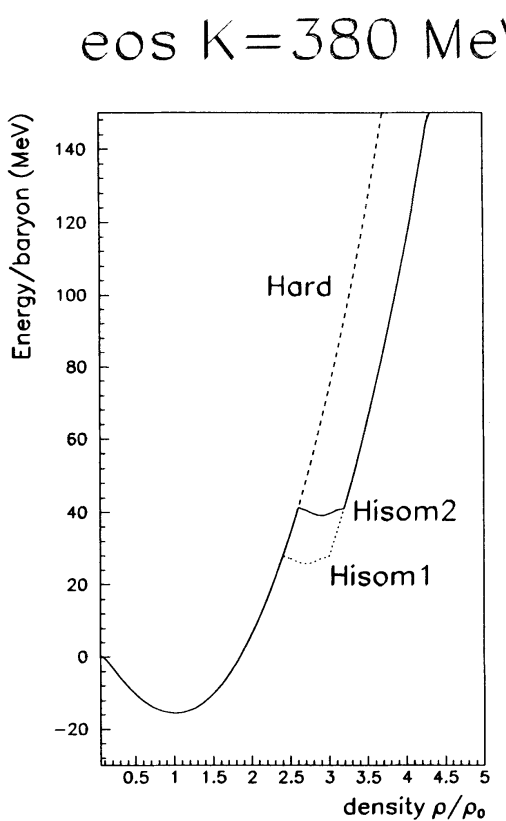

FIG. 1. The equations of state (at zero temperature) employed in our calculation.

high density allows the system to reach a higher density and hence a shorter mean free path and therefore favors the production of kaons. If a density isomer exists there is a region at which there is no additional energy necessary to compress the system further. If the system enters this region in the course of a heavy ion reaction we expect a rather sudden increase of the density and consequently an increase of the kaons as well. Thus all depends on whether the system reaches the required density. Below that density the system does not "notice" the fact that an isomer exists. Since the density is a monotonous function of the energy, we expect a jump to appear in the excitation function. Because at the same beam energy the momentum dependent potentials yield a smaller density, we would expect that there the jump in the excitation function takes place at a higher beam energy. Because of the increasing number of inelastic collisions, we expect also a moderate dependence of the pion yield.

The transverse momentum transfer, on the other hand, is caused by the potential gradient, which is built up during the collision between the high density central region and the surrounding region $[20,21]$. This density gradient is the source of a potential gradient. Particles which suffer a large transverse momentum transfer never enter the high density region but the density gradient which causes a force reflects them sidewards. The average density which these particles "see" is around the normal nuclear matter density. Thus the flow tests the potential around $\rho_{0}$. Consequently the flow analysis yields information about the surface of the high density zone rather than about the compression zone itself. We therefore expect little change if we modify the equation of state at 
densities beyond $2 \rho_{0}$.

Thus kaons and the transverse momentum transfer test the potential energy and therefore the EOS at different densities.

We come now to the numerical results. We performed calculations of the reaction $\mathrm{Au}+\mathrm{Au}, b=3 \mathrm{fm}$ for the six different equations of state employing the BUU/VUU model. The details of this model can be found in Ref. [1]. Figure 2 displays, from top to bottom, the number of collisions, the kaon, and the pion yield. Up to $600 \mathrm{MeV}$ the number of collisions is the same for all static, respectively, for all momentum dependent EOS's. At $700 \mathrm{MeV}$ Hisom 1 starts to deviate from $\mathrm{H}$ and Hisom 2. At this energy the system reaches densities more than $\rho / \rho_{0}=2.4$ but not yet a density of $2.6 \rho / \rho_{0}$. If the density of $\rho / \rho_{0}=2.4$ is reached for Hisom 1 we have a negative pressure and the system collapses. Hence the mean free path decreases and as a consequence the number of collisions increases. At $900 \mathrm{MeV}$ we also observe a difference between Hisom 2 and $\mathrm{H}$. At that energy the system exceeds the density of $2.6 \rho / \rho_{0}$ and hence the density isomer of Hisom 2 becomes effective. At still higher densities the exact position of the isomer becomes unimportant and the number of collisions coincides for Hisom 1 and Hisom 2. The density reached for the EOS's with density isomers is about $0.6 \rho_{0}$ larger than for the hard EOS. Thus the compressional energy stays about constant. The momentum dependent EOS's follow the same trend, but at an energy which is about $200 \mathrm{MeV}$ higher.

At $600 \mathrm{MeV} /$ nucleon the kaon yield of all three EOS's of the same type is identical. At that energy the density of the system does not exceed $\rho / \rho_{0}=2.4$. At $E_{\text {beam }}$ equal to $700 \mathrm{MeV} /$ nucleon the kaon yield for Hisom 1 is more than 1 order of magnitude higher than that of the other two. The density of $\rho / \rho_{0}=2.4$ is reached around $E_{\text {beam }}$ $=1000 \mathrm{MeV} /$ nucleon and consequently we now observe the jump in the kaon yield for Hisom 2. At higher energies the difference between Hisom 1 and Hisom 2 becomes smaller because the density is now beyond $3.2 \rho / \rho_{0}$ where both EOS's become identical. Also the difference between the EOS's with isomer and the hard EOS becomes smaller. More and more kaons are produced then in peripheral reactions because the Fermi motion can provide the additional energy to overcome the threshold of $1.58 \mathrm{GeV}$. At still higher energies we expect that the majority of the kaons come from these peripheral reactions. Therefore the three curves merge and kaons are not suited anymore as a signal of a density isomer. However, if the isomer appears at a density which is not reached with beam energies below the kaon threshold, other processes with a higher threshold may be used, like the production of $\bar{p}$ 's or $\bar{\Lambda}$ 's. The momentum dependent EOS's follow the same trend but once more shifted by $200 \mathrm{MeV}$. The jump of the excitation function is not that pronounced but is still about 1 order of magnitude, and therefore easy to measure. As already explained in Ref. [4] the kaon yield of the momentum dependent interactions is always lower

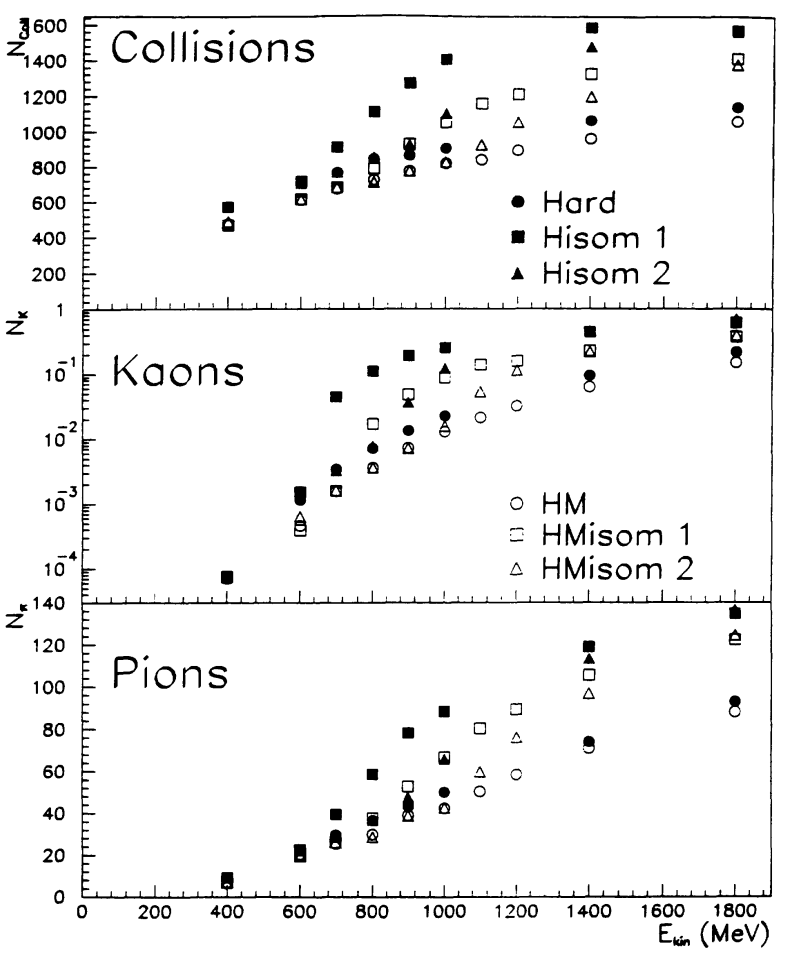

FIG. 2. The number of collisions produced kaons, and pions as a function of the beam energy for $\mathrm{Au}+\mathrm{Au}, b=3 \mathrm{fm}$ for the six equations of state.

as compared to their static counterparts.

The pions show an intermediate behavior. From the enhanced kaon production we can conclude an enhanced $\Delta$ production in the high density region. However, only very few of the observed pions come directly from the high density zone. Most of them are reabsorbed on their way to the surface and the majority of the observed pions are produced close to the surface of the system. However, their number still changes by $40 \%$ if a density isomer is present.

Figure 3 presents the results for three dynamical observables: the flow measured by $p_{x}^{\text {dir }}=\sum_{i=1}^{N} \operatorname{sgn}\left(y_{i}\right)$ $\times p_{x}(i)$, the transverse energy $E_{T} / N=\sqrt{m^{2}+p_{T}^{2}}-m$, and $\sigma\left(p_{z}\right)=\sqrt{\left\langle p_{z}^{2}\right\rangle-\left\langle p_{z}\right\rangle^{2}}$. When the strong increase of the particle production takes place we observe practically no difference of the flow between HM (hard equation of state with momentum dependence) and HMisom (hard equation of state with a density isomer and momentum dependence). Only at much higher energies do we see, as already anticipated [22-25], a reduction of the flow. There the zone of negative pressure has increased to that extent that it starts to have influence on the potential gradient in the low density zone-where the flow is produced.

The transverse energy increases for HMisom 1 and HMisom 2 as a consequence of the higher stopping and the subsequent thermalization caused by the increase of the number of collisions. The same is seen in the direction of the beam where $\sigma\left(p_{z}\right)$ has also increased. A 


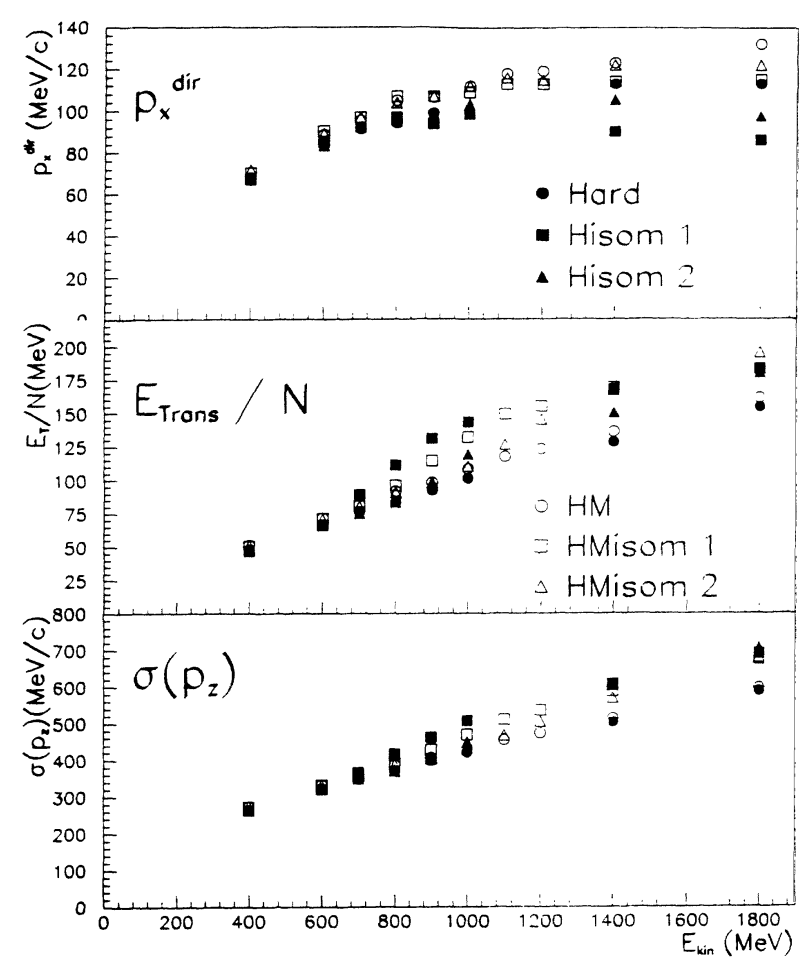

FIG. 3. The flow, the transverse energy, and $\sigma\left(p_{z}\right)$ as a function of the beam energy for $\mathrm{Au}+\mathrm{Au}, b=3 \mathrm{fm}$ for the six equations of state.

detailed investigation shows that this increase is not due to less stopping but to more thermalization.

In conclusion, if a density isomer is present, it will have strong influence on several observables, especially the particle production yields, whereas other observables change little. Hence the puzzling experimental observation may point to the fact that the three parameter parametrization (with all parameters adjusted to nuclear matter properties at $\rho_{0}$ ) does not give sufficient freedom to find a potential which reproduces the experiment at low and high densities.

Is there any evidence for the existence of an isomer yet? Not really, but we would like to point out one remarkable fact. Harris et al. [26] have measured the excitation function of the $\pi^{-}$production and for the systems La $+\mathrm{La}$ and $\mathrm{Ar}+\mathrm{KCl}$. If we take the ratio of the observed pion yield $R=N_{\pi}(1800 \mathrm{MeV}) / N_{\pi}(600 \mathrm{MeV})=7$ and compare it with our calculation we find the values of 4.29 and 6 for $\mathrm{H}$ and Hisom. However, this can only be a hint; a quantitative comparison would require an impact parameter averaging.

Theoretical calculations conjecture the density isomers at densities larger than $2.5 \rho_{0}$. At $1 \mathrm{GeV} /$ nucleon, the highest energy available at SIS, we are just at the bound- ary of this domain. Thus it would be interesting to be able to measure this excitation function up to the AGS energies, where an enhancement of the kaon production has been observed as well.

[1] H. Stöcker and W. Greiner, Phys. Rep. 137, 277 (1986).

[2] W. Cassing et al., Phys. Rep. 188, 361 (1990).

[3] J. Aichelin, Phys. Rep. 202, 233 (1991).

[4] J. Aichelin et al. Phys. Rev. Lett. 58, 1926 (1987).

[5] E. Feenberg and H. Primakoff, Phys. Rev. 70, 980 (1946); A.R. Bodmer, Phys. Rev. D 4, 1601 (1971); A.B. Migdal, Sov. Phys. JETP 34, 1184 (1972); T.D. Lee and G.C. Wick, Phys. Rev. D 9, 2291 (1974); W. Weise and G.E. Brown, Phys. Rep. 27, 1 (1976).

[6] J.D. Walecka, Ann. Phys. (N.Y.) 83, 491 (1974).

[7] J. Boguta and A.R. Bodmer, Nucl. Phys. A292, 413 (1977).

[8] M. Rufa, P.G. Reinhard, J.A. Maruhn, W. Greiner, and M.R. Strayer, Phys. Rev. C 38, 390 (1988).

[9] J. Boguta, Phys. Lett. 109B, 251 (1982); J. Boguta and H. Stöcker, Phys. Lett. 120B, 289 (1983).

[10] B. Waldhauser, J.A. Maruhn, H. Stöcker, and W. Greiner, Z. Phys. A 328, 19 (1987).

[11] Th. Wienold, GSI Report No. 93-28 (unpublished).

[12] E. Grosse, Prog. Nucl. Part. Phys. 30, 89 (1993).

[13] W. Ahner, GSI Report No. 93-29 (unpublished).

[14] C.M. Ko, X.S. Fang, and Y.M Zheng, in Proceedings of the International Workshop on Gross Properties of $\mathrm{Nu}$ clei and Nuclear Excitations XXI, Hirschegg (Austria), 1993, edited by H. Feldmeier (TH Darmstadt, Germany, 1993), p. 29; X.S. Fang et al., Phys. Rev. C 49, R608 (1994).

[15] A. Lang et al., Nucl. Phys. A541, 507 (1992); T. Maruyama et al. (to be published).

[16] C. Hartnack et al., Nucl. Phys. (to be published); J. Aichelin and C.M. Ko, Phys. Rev. Lett. 55, 2661 (1985).

[17] C. Hartnack, GSI Report No. 93-05 (unpublished); (to be published).

[18] J. Aichelin and C.M. Ko, Phys. Rev. Lett. 55, 2661 (1985).

[19] L. Xiong, C.M. Ko, and J.P. Wu, Phys. Rev. C 42, 2231 (1990).

[20] C. Hartnack et al., in Proceedings of the NATO Advanced Course on the Nuclear Equation of State, NATO ASI Ser. B, Vol. 216A (Plenum, New York, 1990), p. 239.

[21] J. Jänicke and J. Aichelin, Nucl. Phys. A547, 542 (1992).

[22] H.G. Baumgardt et al., Z. Phys. A 273, 359 (1975).

[23] J. Hoffmann, H. Stöcker, U. Heinz, W. Scheid, and W. Greiner, Phys. Rev. Lett. 36, 88 (1976).

[24] H. Stöcker, W. Greiner, and W. Scheid, Z. Phys. A 286 , 121 (1978).

[25] H. Stöcker, J. Maruhn, and W. Greiner, Phys. Lett 81B, 303 (1979).

[26] J. W. Harris et al., Phys. Rev. Lett. 58, 463 (1987). 